Research Article

\title{
Relative Position Model Predictive Control of Double Cube Test-Masses Drag-Free Satellite with Extended Sliding Mode Observer
}

\author{
Enyou Wang $\left(\mathbb{D}^{1},{ }^{1}\right.$ Jinxiu Zhang, ${ }^{1,2}$ Huayi Li, ${ }^{1}$ and Ming Liu ${ }^{1}$ \\ ${ }^{1}$ School of Astronautics, Harbin Institute of Technology, Harbin 150001, China \\ ${ }^{2}$ School of Aeronautics and Astronautics, Sun Yat-sen University, Guangzhou 511436, China \\ Correspondence should be addressed to Enyou Wang; enyou.w@gmail.com
}

Received 11 September 2020; Revised 26 November 2020; Accepted 13 December 2020; Published 23 January 2021

Academic Editor: Shihong Ding

Copyright (C) 2021 Enyou Wang et al. This is an open access article distributed under the Creative Commons Attribution License, which permits unrestricted use, distribution, and reproduction in any medium, provided the original work is properly cited.

\begin{abstract}
The drag-free satellites, being space-borne ultrahigh precise measurement platforms, have played irreplaceable roles in a great number of space science missions such as navigation, earth science, fundamental physics, and astrophysics. Most of these missions have to be performed based on the satellites placed with double cube test-masses, which makes the satellite layout and control strategy be more complex. This paper investigates the orbit keeping control problem of a class of low Earth orbit drag-free satellites with double cube test masses. A disturbance observer-based composite control method is proposed, which consists of an extended sliding mode observer and the tube-based robust model predictive control approach. In this design, the observer is proposed to estimate the relative position and velocity of the satellite and the external space disturbance force. A tube-based robust model predictive control scheme is then developed to stabilize the satellite orbit control systems in the presence of actuator saturation, state constraints, and additive stochastic noises. Finally, a simulation example is presented to demonstrate the efficacy and superiority of the proposed orbit control method.
\end{abstract}

\section{Introduction}

In recent years, the drag-free satellites [1], being space-borne ultrahigh precise measurement platforms, have played irreplaceable roles in many space science missions, such as the test of equivalence principle [2], the measurement of the Earth gravity field [3], and the detection of gravitational waves [4]. The drag-free satellites possess many advantages; for example, they can provide autonomous precision orbit determination, map the static and time-varying components of the Earth's mass distribution more accurately, deepen the understanding of the fundamental force of gravity, eventually open up a new window to the universe through the detection and observation of gravitational waves, and so forth.

The key technology of the drag-free satellite is the gravitational reference sensor (GRS), which insulates an internal free-floating test mass (TM, also called proof mass) from both external disturbances and disturbances caused by the spacecraft itself [5]. The drag-free satellites can be divided into two types [6]. The first one is the "accelerometer" drag-free mode, where an electrostatic accelerometer is used as the primary sensor and an electrostatic suspension actuator is paired to maintain the TM to be centred in its cage; therefore it can counter the disturbance forces acting on the spacecraft [7-11]. The second one is free-falling TM mode, in which the satellite provides indirect drag-free behaviour by tracking the movement of the free-falling TM in the cage [12-15]. In particular, the structure of a satellite with two cube TMs is always regarded as the primary layout in these missions, which makes the GRS be more complex and the control system design work be more challenging. For example, as shown in Figure 1, the drag-free satellite containing two cube TMs will enter a low Earth orbit (LEO), which decays more rapidly due to the decelerating effects of the Earth's atmosphere. 


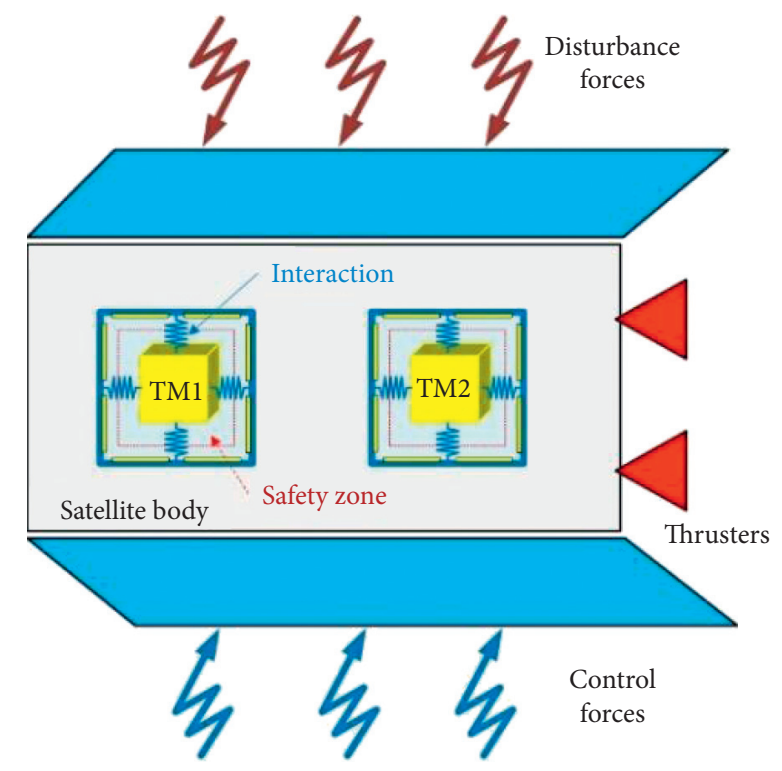

Figure 1: A kind of drag-free satellite structure with double testmasses.

In the research area of control for drag-free satellites, a trade-off should be considered among system complexity, fuel conservation, cost of components, operations, performance, and so forth [16]. In fact, it is always difficult for the drag-free satellite to obtain control satisfactory performance due to the existence of external time-varying environmental disturbances and the unmodelled internal uncertainties. To solve this problem, various types of robust control algorithms have been proposed, such as model predictive control (MPC) [17], $H_{\infty}$ control $[18,19], \mu$-synthesis control [20], embedded model control (EMC) technique [21], and quantitative feedback theory [22]. However, these design results can only realize disturbance attenuation but not disturbance rejection. In order to improve closed-loop control performance, the disturbance observerbased control [23-31] and active disturbance rejection control [32-39] have been developed for drag-free satellite to pursue more ideal control system performance [25, 40-50]. However, how to design a suitable observer to estimate the disturbances more precisely is still an open and attractive problem [51].

This paper investigates the orbit control problem for a class of LEO drag-free satellites with double cube TMs based on the relative position dynamics with state constraints, actuator saturation, and the additive stochastic disturbances. In this design, an extended sliding mode observer method is developed to estimate the system states. Then, a tube-based robust model predictive control (TRMPC) approach is presented [52] to stabilize the resulting orbit control systems. In particular, the TRMPC design approach is divided into the following two steps: (i) an offline evaluation of the constraints to ensure the uncertain future trajectories to lie in sequence of sets, which is called tubes, and (ii) an online MPC scheme designed for the nominal trajectories. As a result, the orbit control issue for the drag-free satellite is solved.

The remaining of this paper is organized as follows. Section 2 formulates the design problem under investigation; the design of disturbance observation-based composite control strategy is presented in Section 3. A demonstrated simulation example is provided in Section 4 , and the paper is concluded in Section 5.

\section{Problem Formulation}

According to $[22,53]$, for a LEO satellite containing two TMs, as shown in Figure 2, the drag-free control strategy is defined as follows: TM1 is chosen as the gravitational reference, which flies freely in a pure gravitational orbit, and the satellite and TM2 are controlled to follow TM1; then the linearized relative position dynamics between the TMs and the satellite are given as follows:

$$
\ddot{\rho}_{1 i}(t)+M_{t} \dot{\rho}_{1 i}(t)+N_{t} \rho_{1 i}(t)=\frac{1}{m_{i}}\left[F_{c, 1 i}(t)+F_{d, 1 i}^{\prime}(t)\right], \quad i=0 \text { or } 2,
$$

where $i=0$ means the satellite and $i=2$ means the TM2; $\rho_{1 i}(t), m_{i}$, and $F_{c, 1 i}(t)$ represent the relative position variables, the mass of the satellite or the TM2, and the control forces; $F_{d, 1 i}{ }^{\prime}(t)$ is the sum of all kinds of disturbances, which for the satellite, the air drag, the solar radiation pressure, and the thruster quantization error and other stochastic noises is considered primarily, and, for the TM2, it mainly consists of electrostatic interference signals, actuator quantization error, and other stochastic noises $[9,40]$.

$$
\begin{aligned}
M_{t} & =\left[\begin{array}{ccc}
0 & -2 \omega_{0} & 0 \\
2 \omega_{0} & 0 & 0 \\
0 & 0 & 0
\end{array}\right]-K_{t}, \\
N_{t} & =\left[\begin{array}{ccc}
-3 \omega_{0} & 0 & 0 \\
0 & 0 & 0 \\
0 & 0 & \omega_{0}^{2}
\end{array}\right]-D_{t},
\end{aligned}
$$

where $\omega_{0}$ is orbit angular velocity of the TM1 and $K_{t}$ and $D_{t}$ are the damping factors between the TM and its condenser cage [54].

The desired positions and velocities of the satellite and TM2 are denoted as $\rho_{10}^{d}(t), \dot{\rho}_{10}^{d}(t), \rho_{12}^{d}(t)$, and $\dot{\rho}_{12}^{d}(t)$, respectively. The desired positions $\rho_{10}^{d}(t)$ and $\rho_{12}^{d}(t)$ are some constants dependent on the layout of the satellite. The relative velocities are defined as follows: $\dot{\rho}_{10}^{d}(t)=0$ and $\dot{\rho}_{12}^{d}(t)=0$. Define the following error variables for case of presentation:

$$
\begin{array}{r}
\tilde{\rho}_{1 i}(t)=\rho_{1 i}(t)-\rho_{1 i}^{d}(t), \\
\dot{\tilde{\rho}}_{1 i}(t)=\dot{\rho}_{1 i}(t)-\dot{\rho}_{1 i}^{d}(t), \\
i=0 \text { or } 2 .
\end{array}
$$

By substituting (3) into (1), one can obtain

$$
\ddot{\tilde{\rho}}_{1 i}(t)+M_{t} \dot{\tilde{\rho}}_{1 i}(t)+N_{t} \tilde{\rho}_{1 i}(t)=\frac{1}{m_{i}}\left[F_{c, 1 i}(t)+F_{d, 1 i}(t)\right], \quad i=0 \text { or } 2 .
$$

Due to the limit volume of the capacitor cage, the relative error variables should satisfy $\widetilde{\rho}_{1 i}(t) \leq \widetilde{\rho}_{\max }$ and $\dot{\tilde{\rho}}_{1 i}(t) \leq \dot{\tilde{\rho}}_{\max }$, which are the state constraints. For convenience, formations (4) should be translated into a state space model. By 


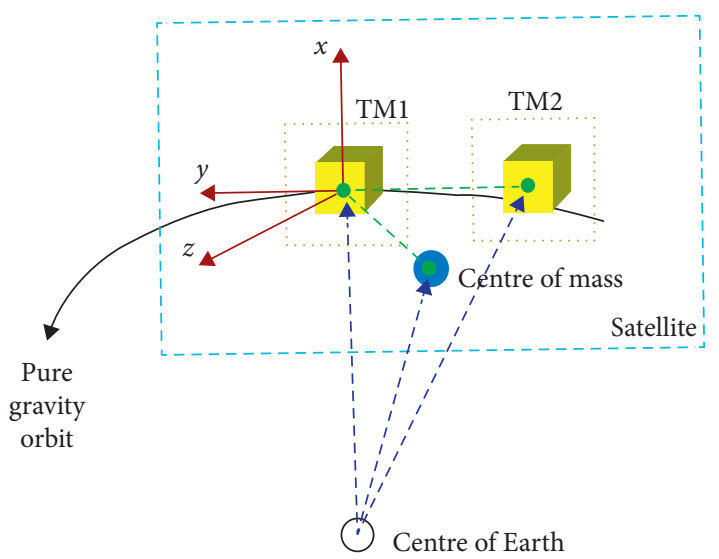

FIgURE 2: The relative position dynamics between the TMs and the satellite, with respect to TM1 Hill reference system.

choosing $\quad x_{1}(t)=\left[\tilde{\rho}_{10}^{T}(t), \dot{\tilde{\rho}}_{10}^{T}(t)\right]^{T} \quad$ and $\quad x_{2}(t)=\left[\tilde{\rho}_{12}^{T}(t)\right.$, $\left.\dot{\tilde{\rho}}_{12}^{T}(t)\right]^{T}, u_{1}(t)=F_{c, 10}(t), u_{2}(t)=F_{c, 12}(t), F_{d, 10}(t)=\omega_{1}(t)+$ $\xi_{1}(t)$, and $F_{d, 12}(t)=\omega_{2}(t)+\xi_{2}(t)$, where $\omega_{i}(t)$ and $\xi_{i}(t)(i=1,2)$ denote the unknown stochastic noises and unknown estimable disturbances, respectively. Then, the state space model is given as follows:

$$
\left\{\begin{array}{l}
\dot{x}_{i}(t)=A_{i} x_{i}(t)+B_{i} u_{i}(t)+B_{\omega, i}\left(\omega_{i}(t)+\xi_{i}(t)\right), \\
y_{i}(t)=C_{i} x_{i}(t)
\end{array}\right.
$$

where

$$
\begin{aligned}
A_{i} & =\left[\begin{array}{ll}
0_{3} & I_{3} \\
N_{t} & M_{t}
\end{array}\right], \\
B_{1} & =B_{\omega, 1}=\left[\begin{array}{c}
0_{3} \\
\frac{1}{m_{0}} I_{3}
\end{array}\right], \\
B_{2} & =B_{\omega, 2}=\left[\begin{array}{c}
0_{3} \\
\frac{1}{m_{2}} I_{3}
\end{array}\right], \\
C_{i} & =\left[\begin{array}{ll}
I_{3} & 0_{3}
\end{array}\right], \\
i & =1,2 .
\end{aligned}
$$

Before designing the disturbance observer-based composite control strategy, the following assumptions are given about $\omega_{i}(\mathrm{t})$ and $\xi_{i}(t)(i=1,2)$.

Assumption 1. The unknown external disturbance $\xi_{i}(t)(i=$ 1,2) satisfies the following: $\left\|\xi_{i}(t)\right\|<\varrho_{i}$ and $\left\|\dot{\xi}_{i}(t)\right\|<\varsigma_{i}$, where $\varrho_{i}$ and $\varsigma_{i}$ are known real constants.

Assumption 2. The unknown stochastic noise $\omega_{i}(t)(i=1,2)$ is assumed to be discontinuous but bounded subject to $\left\|\omega_{i}(t)\right\| \leq \omega_{i}$ and belongs to a bounded and convex subset $\mathbb{W} \subset \mathbb{R}^{n}$ containing the origin in its interior.

\section{Design of Disturbance Observer-Based Composite Control}

The control system composition of a LEO satellite with two cube TMs is shown in Figure 3. To achieve the "drag-free" goal and eliminate the effects of external disturbances (the environment disturbances may contain the atmosphere and the solar radiation pressure, the thruster quantization error, the electrostatic noises, the actuator quantization error, etc.), a disturbance observer-based composite control approach is proposed, which consists of an extended sliding mode observer and a tube-based robust model predictive control. The detailed design process is as follows. First, for system (5), the observer is proposed to estimate states $x_{i}$ for state feedback control and $\xi_{i}(t)$ for active disturbance rejection control $u_{f f c, i}$. Second, a tube-based robust model predictive control $u_{i}=u_{f b c, i}+u_{f f c, i}$ is adopted, which can cope with state constraints and actuator saturation and attenuate the effects of additive stochastic noises. For convenience, in the following discussion, the subscript $i$ in (5) is omitted.

3.1. Design of Extended Sliding Mode Observers. Motivated by the augmented strategy method in $[34,36,55]$, we define the following extended vectors and matrices:

$$
\begin{aligned}
\bar{x}(t) & =\left[x^{T}(t), \xi^{T}(t)\right]^{T}, \\
\bar{A} & =\left[\begin{array}{cc}
A & B_{\omega} \\
0 & 0
\end{array}\right], \\
\bar{B} & =\left[\begin{array}{l}
B \\
0
\end{array}\right], \\
\bar{D} & =\left[\begin{array}{l}
0 \\
I_{p}
\end{array}\right], \\
\bar{B}_{w} & =\left[\begin{array}{c}
B_{w} \\
0
\end{array}\right], \\
\bar{C} & =\left[\begin{array}{ll}
C & 0
\end{array}\right] .
\end{aligned}
$$

For system (7), consider the following continuous-time extended SMO [55]:

$$
\dot{\overline{\hat{x}}}(t)=\bar{A} \hat{\bar{x}}(t)+\bar{B} u(t)+L \tilde{y}(t)+\bar{D} F v_{2}(t),
$$

where $\tilde{y}(t)=y(t)-\bar{C} \hat{\bar{x}}(t)$ and $v_{2}(t)$ is the discontinuous term designed as follows:

$$
v_{2}(t)= \begin{cases}\eta \frac{\tilde{y}(t)}{\|\widetilde{y}(t)\|}, & \text { if }\|\widetilde{y}(t)\|>\frac{\varepsilon}{\eta} \\ \eta^{2} \frac{\tilde{y}(t)}{\varepsilon}, & \text { if }\|\widetilde{y}(t)\| \leq \frac{\varepsilon}{\eta}\end{cases}
$$

where $\eta>\left(\bar{\lambda}_{F} / \underline{\lambda}_{F}^{2}\right)$ and $\bar{\lambda}_{F}=\sqrt{\lambda_{\max }\left(F^{T} F\right)}, \lambda_{F}=\sqrt{\lambda_{\min }\left(F^{T} F\right)}$, and $\lambda_{\max }\left(F^{T} F\right)$ and $\lambda_{\min }\left(F^{T} F\right)$ are the maximal and minimal nonzero eigenvalues of matrix $F^{T} F$, respectively. 


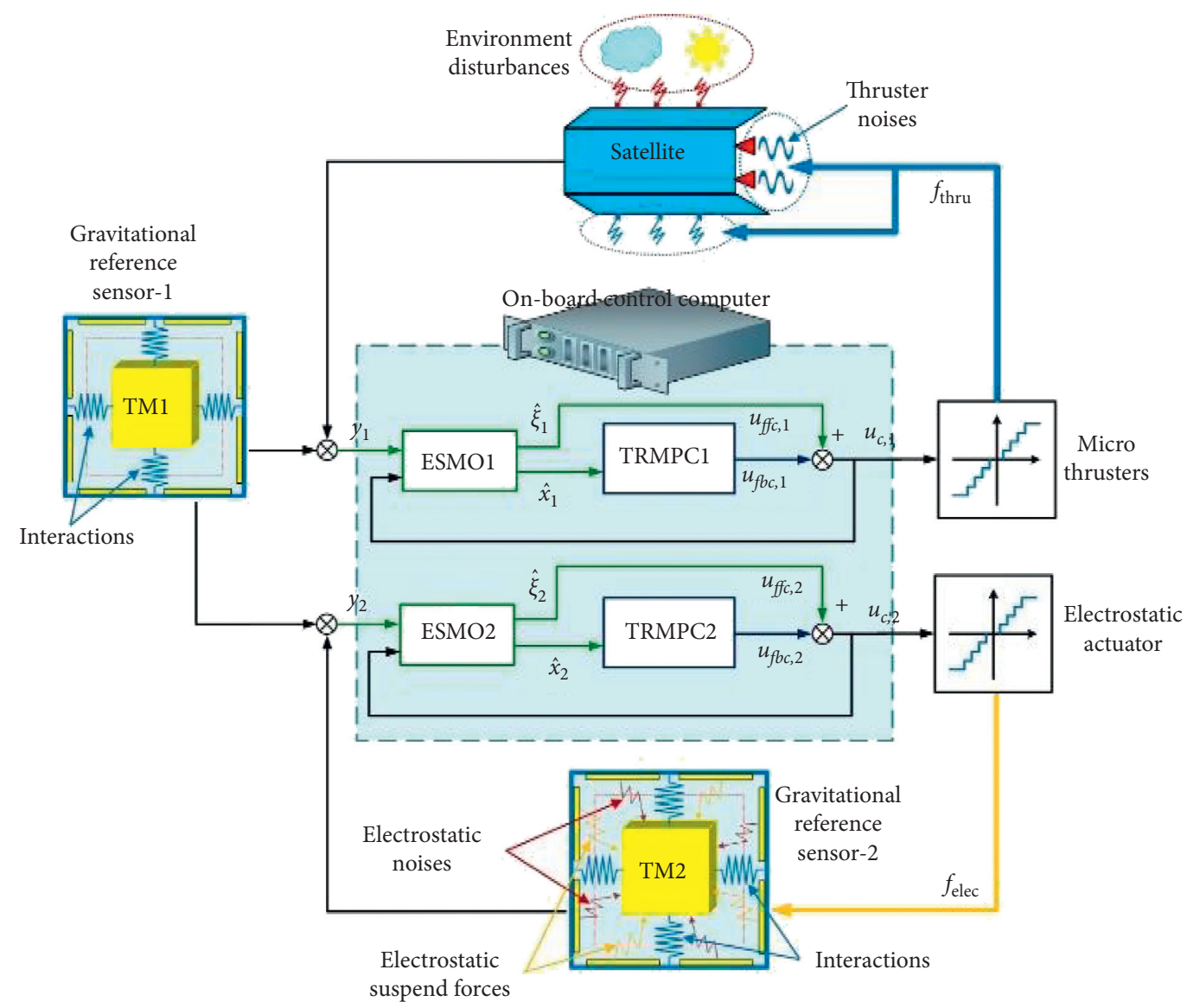

FIGURE 3: The control system schematic diagram of a LEO satellite with two cube TMs; the satellite and TM2 are controlled, respectively.

Remark 1. It is worth mentioning that the main function of the proposed extended SMO is to estimate the state and disturbances vectors simultaneously, which is a design basis of the subsequent composite control law.

Define $\widetilde{\bar{x}}(t)=\bar{x}(t)-\widehat{\bar{x}}(t)$; then the error system is derived as follows:

$$
\dot{\bar{x}}(t)=(\bar{A}-L \bar{C}) \tilde{\bar{x}}(t)+\bar{D}\left(\dot{\xi}(t)-F v_{2}(t)\right)+\bar{B}_{w} \omega(t) .
$$

Lemma 1 (see [56]). System (5) has the relative degree $n$ with respect to the unknown input $\xi(t)$ (i.e., the system is strongly observable).

Lemma 2. If the matrix pair $(A, C)$ is detectable and the condition

$$
\operatorname{rank}\left(\left[\begin{array}{cc}
A & B_{\omega} \\
C & 0
\end{array}\right]\right)=n+p
$$

holds, then the pair $(\bar{A}, \bar{C})$ is detectable; that is, there exists an observer gain matrix $L$ such that $\bar{A}-L \bar{C}$ is Hurwitz.

Proof. It can be derived that

$$
\operatorname{rank}\left(\left[\begin{array}{c}
\bar{A}-\lambda I_{n+p} \\
\bar{C}
\end{array}\right]\right)=\operatorname{rank}\left(\left[\begin{array}{cc}
A-\lambda I_{n} & B_{w} \\
0_{p \times n} & -\lambda I_{p} \\
C & 0_{q \times p}
\end{array}\right]\right) .
$$

Case 1 . When $\lambda \neq 0$, for the matrix in right side of equation (12), we have

$$
\left[\begin{array}{ccc}
I_{n} & \lambda^{-1} B_{w} & 0 \\
0 & I_{p} & 0 \\
0 & 0 & I_{q}
\end{array}\right]\left[\begin{array}{cc}
A-\lambda I_{n} & B_{w} \\
0_{p \times n} & -\lambda I_{p} \\
C & 0_{q \times p}
\end{array}\right]=\left[\begin{array}{cc}
A-\lambda I_{n} & 0_{p \times n} \\
0_{p \times n} & -\lambda I_{p} \\
C & 0_{q \times p}
\end{array}\right],
$$


which means that

$$
\begin{aligned}
\operatorname{rank}\left(\left[\begin{array}{cc}
A-\lambda I_{n} & B_{w} \\
0_{p \times n} & -\lambda I_{p} \\
C & 0_{q \times p}
\end{array}\right]\right) & =\operatorname{rank}\left(\left[\begin{array}{ccc}
I_{n} & \lambda^{-1} B_{w} & 0 \\
0 & I_{p} & 0 \\
0 & 0 & I_{q}
\end{array}\right]\left[\begin{array}{cc}
A-\lambda I_{n} & B_{w} \\
0_{p \times n} & -\lambda I_{p} \\
C & 0_{q \times p}
\end{array}\right]\right) \\
& =\operatorname{rank}\left(\left[\begin{array}{cc}
A-\lambda I_{n} & 0_{n \times p} \\
0_{p \times n} & -\lambda I_{p} \\
C & 0_{q \times p}
\end{array}\right]\right) \\
& =\operatorname{rank}\left(\left[\begin{array}{c}
A-\lambda I_{n} \\
\bar{C}
\end{array}\right]\right)+p .
\end{aligned}
$$

That means

$$
\operatorname{rank}\left(\left[\begin{array}{c}
\bar{A}-\lambda I_{n+p} \\
\bar{C}
\end{array}\right]\right)=\operatorname{rank}\left(\left[\begin{array}{c}
A-\lambda I_{n} \\
\bar{C}
\end{array}\right]\right)+p .
$$

Hence, if the pair $(A, C)$ is detectable, we have

$$
\operatorname{rank}\left(\left[\begin{array}{c}
A-\lambda I_{n} \\
\bar{C}
\end{array}\right]\right)=n .
$$

Then, the following rank condition holds:

$$
\operatorname{rank}\left(\left[\begin{array}{c}
A-\lambda I_{n+p} \\
\bar{C}
\end{array}\right]\right)=n+p
$$

Case 2. When $\lambda=0$, if rank condition (11) holds, we have

$$
\operatorname{rank}\left(\left[\begin{array}{c}
\bar{A} \\
\bar{C}
\end{array}\right]\right)=\operatorname{rank}\left(\left[\begin{array}{cc}
A & B_{w} \\
C & 0
\end{array}\right]\right)=n+p
$$

Finally, for both cases, we can always imply $\operatorname{rank}\left(\left[\begin{array}{c}A-\lambda I_{n+p} \\ \bar{C}\end{array}\right]\right)=n+p, \lambda \in \mathbb{C}, \operatorname{Re}[\lambda] \geq 0$, which implies that the pair $(\bar{A}, \bar{C})$ is detectable.

To attenuate the influence of $\omega(t)$ for error system (10), we define the prescribed $H_{\infty}$ performance index as follows:

$$
\|H\|_{\infty}=\sup _{\|\omega(t)\|_{L_{2}} \neq 0} \frac{\|\psi \tilde{\bar{x}}(t)\|_{L_{2}}}{\|\omega(t)\|_{L_{2}}} \leq \sqrt{\gamma_{1}},
$$

where $\psi \tilde{\bar{x}}(t)$ is the weighted estimation error and $\psi$ is the weight matrix. The following main theorem provides the existence condition of the proposed observer (8).

Theorem 1. The solution of observer error system (10) is asymptotically stable with the prescribed $H_{\infty}$ performance index if there exist positive and definite matrix $Q>0$ and matrix $F$ with appropriate dimensions, in minimizing $\gamma_{1}>0$, such that the following matrix constraints hold:

$$
\begin{aligned}
\bar{Q} & =\left[\begin{array}{cc}
\bar{A}^{T} Q+Q \bar{A}-\bar{C}^{T} \bar{C}+\psi^{T} \psi & Q \bar{B}_{w} \\
\bar{B}_{w}^{T} Q & -\gamma_{1} I
\end{array}\right]<0, \\
Q \bar{D} & =\bar{C}^{T} F^{T} .
\end{aligned}
$$

Moreover, the observer gain is designed as $L=(1 / 2) Q^{-1} \bar{C}^{T}$.

Proof. For system (10), select the Lyapunov function as $V(t)=\widetilde{\bar{x}}^{T}(t) Q \widetilde{\bar{x}}(t)$ with $Q>0$ being designed; it is easy to obtain that

$$
\begin{aligned}
\dot{V}(t) & =\dot{\overline{\bar{x}}}^{T}(t) Q \widetilde{\bar{x}}(t)+\tilde{\bar{x}}^{T}(t) Q \dot{\overline{\bar{x}}}(t) \\
& =2 \widetilde{\bar{x}}^{T}(t) Q\left[(\bar{A}-L \bar{C}) \widetilde{\bar{x}}(t)+\bar{D}\left(\dot{\xi}(t)-F v_{2}(t)\right)+\bar{B}_{w} \omega(t)\right] \\
& =\widetilde{\bar{x}}^{T}(t)\left[\bar{A}^{T} Q+Q \bar{A}-\bar{C}^{T} \bar{C}\right] \widetilde{\bar{x}}(t)+2 \widetilde{\bar{x}}(t) \bar{C}^{T} F^{T}\left(\dot{\xi}(t)-F v_{2}(t)\right)+\widetilde{\bar{x}}^{T}(t) Q \bar{B}_{w} \omega(t)+\omega^{T}(t) \bar{B}_{w}^{T} Q \widetilde{\bar{x}}(t) \\
& =\widetilde{\bar{x}}^{T}(t)\left[\bar{A}^{T} Q+Q \bar{A}-\bar{C}^{T} \bar{C}\right] \widetilde{\bar{x}}(t)+2 \widetilde{y} F^{T}\left(\dot{\xi}(t)-F v_{2}(t)\right)+2 \widetilde{\bar{x}}^{T}(t) Q \bar{B}_{w} \omega(t)
\end{aligned}
$$


In the following analysis, let $Y(t)=2 \widetilde{y} F^{T}\left(\dot{\xi}(t)-F v_{2}(t)\right)$; we consider two cases separately.

Case 3. $\|\tilde{y}\|>(\varepsilon / \eta)$; in this case, we have

$$
\begin{aligned}
Y(t) & =2 \widetilde{y} F^{T}\left(\dot{\xi}(t)-F \eta \frac{\tilde{y}(t)}{\|\widetilde{y}(t)\|}\right) \\
& \leq 2\|\tilde{y}\|\left(\|F\| \rho-\|F\|^{2} \eta\right) \\
& \leq 2\|\tilde{y}\|\left(\bar{\lambda}_{F} \rho-\underline{\lambda}_{F}^{2} \eta\right) \leq 0,
\end{aligned}
$$

where $\eta$ can be chosen such that $\eta>\left(\bar{\lambda}_{F} / \underline{\lambda}_{F}^{2}\right) \rho$.

Case 4. $\|\tilde{y}\| \leq(\varepsilon / \eta)$; in this case, it can be derived that

$$
\begin{aligned}
Y(t) & =2 \widetilde{y} F^{T}\left(\dot{\xi}(t)-F \eta^{2} \frac{\tilde{y}(t)}{\varepsilon}\right) \\
& \leq 2 \rho \bar{\lambda}_{F}\|\tilde{y}\|-2 \eta^{2} \underline{\lambda}_{F}^{2} \frac{\|\widetilde{y}\|^{2}}{\varepsilon} \leq 2 \eta \underline{\lambda}_{F}^{2}\|\tilde{y}\|-2 \eta^{2} \underline{\lambda}_{F}^{2} \frac{\|\widetilde{y}\|^{2}}{\varepsilon} \leq-2 \underline{\lambda}_{F}^{2}\left(\frac{\eta\|\tilde{y}\|}{\sqrt{\varepsilon}}-\frac{\sqrt{\varepsilon}}{2}\right)^{2}+\frac{\lambda_{F}^{2} \varepsilon}{2} \leq 2 \underline{\lambda}_{F}^{2}\left(\frac{\eta(\varepsilon / \eta)}{\sqrt{\varepsilon}}-\frac{\sqrt{\varepsilon}}{2}\right)^{2}+\frac{\lambda_{F}^{2} \varepsilon}{2} \leq 0 .
\end{aligned}
$$

Based on Cases 3 and 4, we can conclude that

$$
\begin{aligned}
\dot{V}(t) & =Y(t)+\tilde{\bar{x}}^{T}(t)\left[\bar{A}^{T} Q+Q \bar{A}-\bar{C}^{T} \bar{C}\right] \tilde{\bar{x}}(t)+2 \widetilde{\bar{x}}^{T}(t) Q \bar{B}_{w} \omega(t) \\
& \leq \widetilde{\bar{x}}^{T}(t)\left[\bar{A}^{T} Q+Q \bar{A}-\bar{C}^{T} \bar{C}\right] \tilde{\bar{x}}(t)+2 \widetilde{\bar{x}}^{T}(t) Q \bar{B}_{w} \omega(t) .
\end{aligned}
$$

To minimize the effect of the disturbance on the estimation error in the sense of $L_{2}$ norm, we consider the following constraint:

$$
W(t)=\dot{V}(t)+\tilde{\bar{x}}^{T}(t) \psi^{T} \psi \tilde{\bar{x}}(t)-\gamma_{1} \omega^{T}(t) \omega(t) \leq 0 .
$$

In light of Schur complement, it is easy to derive that

$$
\begin{aligned}
& W(t) \leq \tilde{\bar{x}}^{T}(t)\left[\bar{A}^{T} Q+Q \bar{A}-\bar{C}^{T} \bar{C}\right] \tilde{\bar{x}}(t)+\tilde{\bar{x}}^{T}(t) Q \bar{B}_{w} \omega(t)+\omega^{T}(t) \bar{B}_{w}^{T} Q \widetilde{\bar{x}}(t)+\tilde{\bar{x}}^{T}(t) \psi^{T} \psi \widetilde{\bar{x}}^{T}(t)-\gamma_{1} \omega^{T}(t) \omega(t) \\
& \leq\left[\begin{array}{ll}
\tilde{\tilde{x}}^{T}(t) & \omega^{T}(t)
\end{array}\right] \bar{Q}\left[\begin{array}{c}
\tilde{\bar{x}}(t) \\
\omega(t)
\end{array}\right]<0 .
\end{aligned}
$$

Accordingly, it can be seen that $W(t) \leq 0$ is ensured provided that $\bar{Q}<0$ holds. Therefore, the solution of error system (10) is asymptotically stable with the prescribed $H_{\infty}$ performance index as $t \longrightarrow \infty$.

Remark 2. It should be pointed out that, in Theorem 1, the equality constraint $Q \bar{D}=\bar{C}^{T} F^{T}$ can be rewritten as

$$
\operatorname{Trace}\left(\left(Q \bar{D}-\bar{C}^{T} F^{T}\right)^{T}\left(Q \bar{D}-\bar{C}^{T} F^{T}\right)\right)=0 .
$$

Hence, we can introduce the following condition:

$$
\left(Q \bar{D}-\bar{C}^{T} F^{T}\right)^{T}\left(Q \bar{D}-\bar{C}^{T} F^{T}\right)<\gamma_{2} I,
$$

where $\gamma_{2}>0$ is a parameter to be designed. Then the design problem of observer gains $L$ can be converted into the following minimization problem:

$$
\begin{aligned}
& \min \gamma_{1}, \gamma_{2} \\
& \text { subject to (20) and (28). }
\end{aligned}
$$

3.2. Design of Tube-Based Robust Model Predictive Control. For equations (5), $\xi_{i}(t)$ is estimated and eliminated through the extended sliding mode observer; thus we have to compensate it by disturbance compensation feed-forward control. Translate formulations (5) without $\xi_{i}(t)$ into the discrete model of the form in (30) as follows:

$$
x_{k+1}=A_{d} x_{k}+B_{d} u_{k}+B_{\omega_{d}}\left(\xi_{k}+\omega_{k}\right) .
$$

As mentioned before, the controller is designed as $u_{k}=u_{k, f f c}+u_{k, f b c}$, where

$$
u_{k, f f c}=-\widehat{\xi}_{k},
$$

is the feed-forward control part to compensate $\xi_{k}$ and $u_{k, f b c}$ is the model predictive control law to be designed. Define the estimation error as 


$$
\tilde{\xi}_{k}=\widehat{\xi}_{k}-\xi_{k}
$$

Then, system (30) becomes

$$
x_{k+1}=A_{d} x_{k}+B_{d} u_{k, f b c}+B_{\omega_{d}}\left(\omega_{k}-\widetilde{\xi}_{k}\right) .
$$

As proved in the observer design results in Theorem 1, we have $\left\|\widetilde{\xi}_{k}\right\| \longrightarrow 0$ with $k \longrightarrow \infty$; thus $\left\|\omega_{k}-\widetilde{\xi}_{k}\right\| \leq\left\|\omega_{k}\right\|+$ $\left\|\widetilde{\xi}_{k}\right\|=(1+\beta)\left\|\omega_{k}\right\|$ holds, where $\beta>0$ is a small constant and $\beta \longrightarrow 0$ when $k \longrightarrow \infty$.

Define $d_{k}=\omega_{k}-\widetilde{\xi}_{k}$; it satisfies $\left\|d_{k}\right\| \leq(1+\beta) \bowtie$, which belongs to the bounded and convex subset $\mathbb{W}$. Hence, system (30) can be written in the following form:

$$
x_{k+1}=A_{d} x_{k}+B_{d} u_{k, f b c}+B_{\omega_{d}} d_{k} .
$$

The investigated system (30) is subject to hard constraints on both state and input vectors with the following form:

$$
\begin{aligned}
& x \in \mathbb{X}, \\
& u \in \mathbb{U},
\end{aligned}
$$

where $\mathbb{X}$ and $\mathbb{U}$ are polytopes.

To solve this control problem, a robust MPC algorithm is considered [57] by repeatedly solving an optimal control problem, where the finite horizon quadratic cost $J_{N}(x, u)$ to be minimized at the current time $k$ is

$$
J_{N}\left(x_{k}, u_{k}\right)=\sum_{i=0}^{N-1}\left(x_{i \mid k}^{T}\left(2 x_{i \mid k}+u_{i \mid k}^{T} \mathscr{R} u_{i \mid k}\right)+x_{N \mid k}^{T} P x_{N \mid k} .\right.
$$

In (36), $N \in \mathbb{R}^{+}$is the MPC prediction horizon, $\mathbb{Q} \in \mathbb{R}^{n \times n}, \mathscr{Q}>0, \mathscr{R} \in \mathbb{R}^{m \times m}, \mathscr{R}>0$ and $P$ is the solution of the algebraic Riccati equation [57].

$$
\left(A_{d}+B_{d} K\right)^{T} P\left(A_{d}+B_{d} K\right)+\mathscr{Q}+K^{T} \mathscr{R} K=P .
$$

Due to the presence of the unknown disturbance $d_{k}$, we rewrite the state vector $x_{i \mid k}$ of the system as the sum of a nominal part $z_{i \mid k}$ and an error part $e_{i \mid k}$ in the following form:

$$
x_{i \mid k}=z_{i \mid k}+e_{i \mid k},
$$

where $e_{i \mid k}$ denotes the deviation of the real state $e_{i \mid k}$ with respect to the nominal one. (30):

Design the following feedback policy (39) for system

$$
u_{i \mid k}=v_{i \mid k}+K\left(x_{i \mid k}-z_{i \mid k}\right),
$$

where $v_{i \mid k}$ denotes the nominal input vector and the gain matrix $K$ should be selected such that $A_{K}=A_{d}+B_{d} K$ is Schur-stable; then the corresponding nominal and error dynamics can be described, respectively, as follows:

$$
\begin{aligned}
z_{i+1 \mid k} & =A_{d} z_{i \mid k}+B_{d} v_{i \mid k}, \\
z_{0 \mid k} & =x_{0 \mid k}, \\
e_{i+1 \mid k} & =A_{K} e_{i \mid k}+B_{\omega_{d}} d_{i \mid k}, \\
e_{0 \mid k} & =0 .
\end{aligned}
$$

Hence, the finite horizon optimal quadratic cost (36) can be redefined in terms of nominal state $z_{k}$ and control input $v_{k}$ as

$$
J_{N}\left(z_{k}, v_{k}\right)=\sum_{i=0}^{N-1}\left(z_{i \mid k}^{T} \mathscr{Q} z_{i \mid k}+v_{i \mid k}^{T} \mathscr{R} v_{i \mid k}\right)+z_{N \mid k}^{T} P z_{N \mid k},
$$

and the finite horizon optimal control problem can be reformulated as follows.

Definition 1. Given the nominal system dynamics (40), cost (42), and nominal constraints set $\mathbb{Z}, \mathbb{V}, \mathbb{Z}_{f}$, the nominal robust MPC finite horizon optimization problem can be described as

$$
\begin{aligned}
& \min _{v} J_{N}\left(z_{k}, v_{k}\right) \\
& \text { s.t. } \quad z_{i+1 \mid k}=A_{d} z_{i \mid k}+B_{d} v_{i \mid k}, z_{0 \mid k}=x_{k} \\
& z_{i \mid k} \in \mathbb{Z}, \quad i \in[1, N] \\
& v_{i \mid k} \in \mathbb{V}, \quad i \in[0, N-1] \\
& z_{i \mid k} \in \mathbb{Z}_{f} .
\end{aligned}
$$

The solution of (43) is the optimal nominal control sequence $v_{0 \mid k}^{*}=\left[v_{0 \mid k}^{*}\left(0 ; z_{k}\right), \ldots, v_{T-1 \mid k}^{*}\left(T-1 ; z_{k}\right)\right]$ and the first control action, that is, $\widetilde{\kappa}_{N}\left(z_{k}\right):=v_{0 \mid k}^{*}\left(0 ; z_{k}\right)$, represents the optimal control $v_{i \mid k}$ to be applied to system (40).

The proposed control law applied on the uncertain system (40), according to the control policy adopted, is

$$
\begin{aligned}
u_{i \mid k} & =v_{i \mid k}+K\left(x_{i \mid k}-z_{i \mid k}\right)=\kappa_{N}\left(x_{k}, z_{k}\right) \\
& =\widetilde{\kappa}_{N}\left(z_{k}\right)+K\left(x_{k}-z_{k}\right) .
\end{aligned}
$$

The composite closed-loop system then satisfies

$$
\begin{gathered}
x_{i+1 \mid k}=A_{d} x_{i \mid k}+B_{d} \kappa_{N}\left(i, x_{k}, z_{k}\right)+B_{\omega_{d}} d_{i \mid k}, \\
z_{i+1 \mid k}=A_{d} z_{i \mid k}+B_{d} \widetilde{\kappa}_{N}\left(i, z_{k}\right) .
\end{gathered}
$$

For the TRMPC approach, the matrix $K$ in the control policy (39) is designed to stabilize system (30). Consider the following closed-loop system:

$$
x_{i+1 \mid k}=\left(A_{d}+B_{d} K\right) x_{i \mid k}+B_{d} v_{i \mid k}+B_{\omega_{d}} d_{i \mid k} .
$$

Hence, the satisfaction of the following condition aims to define the feedback gain $K$ that stabilizes the system. 


$$
\left(A_{d}+B_{d} K\right)^{T} \widehat{P}\left(A_{d}+B_{d} K\right)-\widehat{P}<0, \quad \widehat{P}>0 .
$$

In order to robustly satisfy the mission constrains, they are tightened to allow the trajectories of the uncertain system, affected by disturbance, to lie in a tube centred on the nominal one, where each trajectory is related to a particular realization of the uncertainty at each time step $k$. The derivations of the nominal state, input, and terminal constrains set $\mathbb{Z}, \mathbb{V}, \mathbb{Z}_{f}$ are described according to the approach proposed in [57], such that the constraints in (39) of system (30) are satisfied for every realization of the disturbance sequence $\omega$ by suitable design of the tube.

We now define $S_{K}(\infty):=A_{K}^{0} \mathbb{W} \oplus A_{K}^{1} \mathbb{W} \oplus \cdots=\sum_{j=0}^{\infty} A_{K}^{j}$ $\mathbb{W}$, where $\oplus$ is the Minkowski sum and $A_{K}^{j} \mathbb{W}:=\left\{A_{K}^{j} d_{k} \mid d_{k}\right.$ $\in \mathbb{W}\}$ is the set multiplication; the uncertain set of the error $e_{i \mid k}$ is the minimal robust positive invariant set for

$$
x_{i+1 \mid k}=A_{d} x_{i \mid k}+B_{\omega_{d}} d_{i \mid k}, \quad d_{k} \in \mathbb{W} .
$$

Then the state and control input vector constraints in (35) are satisfied provided that

$$
\begin{array}{r}
z_{i \mid k} \in \mathbb{X} \Theta S_{K}(\infty), \\
v_{i \mid k} \in \mathbb{U} \Theta K S_{K}(\infty),
\end{array}
$$

where $\Theta$ denotes the Pontryagin set difference. It is obvious that the terminal constraint for system (30) at time instant $N$ is ensured if the normal system (40) satisfies the tighter constraint

$$
z_{N} \in \mathbb{Z} \subseteq \mathbb{X}-S_{K}(\infty), \quad \mathbb{Z}_{f} \subseteq \mathbb{Z} .
$$

Moreover, these assertions only make sense if the disturbance set $\mathbb{W}$ is sufficiently small to satisfy the following Assumption 3, as defined in [57].

Assumption 3. (Restricted disturbances for constraints satisfaction) $S_{K} \subset \mathbb{X}$ and $K \times S_{K} \subset \mathbb{U}$.

The next step is to define a robust positively invariant set $S_{K}$ for [19] to obtain the tighter constraints acting on the nominal system. Then the constraints are considered for the TRMPC problem. Once the uncertainty set $\mathbb{W}$ is evaluated, an inner approximation of the nominal constraint set can be constructed. In this design, we adopt the following strategy presented in [57].

\section{Algorithm 1. Computation of $\mathbb{Z}$ and $\mathbb{V}$}

(1) Define the linear state constraint as: $\mathbb{X}=\left\{x_{i \mid k} \in \mathbb{R}^{n} \mid a x_{i \mid k}<b\right\} ;$

(2) Construct the nominal state constraint inequality $a z_{i \mid k} \leq b-\max \left\{a e_{i \mid k} \mid e_{i \mid k} \in S_{K}(\infty)\right\}=b-\Phi_{\infty} ;$

(3) Approximate the upper value of $\Phi_{\infty}$ as $\Phi_{N}=\max \left\{a \sum_{i=0}^{N-1} A_{K}^{i} d_{i \mid k} \mid d_{i \mid k} \in \mathbb{W}\right\}$

(4) Choose a suitable $\alpha \in(0,1)$ and $N$ such that $A_{K}^{N} d_{i \mid k} \in \alpha \mathbb{W}$, compute $\Phi_{\infty} \leq(1-\alpha)^{-1} \Phi_{N}$

(5) Compute the nominal state constraint set $\mathbb{Z}:=\left\{z_{i \mid k} \in \mathbb{R}^{n} \mid a z_{i \mid k} \leq b-(1-\alpha)^{-1} \Phi_{N}\right\} ;$
(6) Compute the nominal control constraint set $\mathbb{V}:=\left\{v_{i \mid k} \in \mathbb{R}^{m} \mid a^{\prime} v_{i \mid k} \leq b^{\prime}-K(1-\alpha)^{-1} \Phi_{N}\right\}$, where $\mathbb{U}:=\left\{u_{i \mid k} \in \mathbb{R}^{m} \mid a^{\prime} u_{i \mid k} \leq b^{\prime}\right\}$.

Hence, the observer-based model predictive control strategy could be formally described by the following algorithm.

Algorithm 2. Disturbance observer-based model predictive control strategy.

(1) Initialization: at time $k=0$, set $x_{k}=z_{k}=x(0)$ where $x(0)$ denotes the current state.

(2) At time $k$, considering the current state $\left(x_{k}, z_{k}\right)$, based on the disturbance estimation $\widehat{\xi}_{k}$ from the observer (8), solve the nominal optimal control problem (43) to obtain the nominal control vector $v_{k}:=v_{0 \mid k}^{*}\left(0 ; z_{k}\right)$ and the control input vector $u_{k}=v_{k}+K\left(x_{k}-z_{k}\right)-\widehat{\xi}_{k}$.

(3) If the nominal optimal control problem (43) is infeasible, adopt safety/recovery procedure.

(4) Apply the control $u_{k}$ to the system (45) and (46);

(5) Calculate the estimation $\widehat{x}_{k+1}$ from the observer (8) as successor state $x_{k+1}$ of the system (30), and calculate the successor state $z_{k+1}$ of the nominal system (40).

(6) Set $\left(x_{k}, z_{k}\right)=\left(x_{k+1}, z_{k+1}\right)$, set $k=k+1$, and go to (2).

\section{Simulation Results}

In this section, a numerical simulation is carried out to verify the effectiveness of the extended sliding mode-based TRMPC approach. Suppose that the satellite is flying at a little eccentric low Earth orbit with altitude of $300 \mathrm{~km}$. The parameters in (1) are given in Table 1 [54].

For the relative position motion between the TM1 and the satellite, define $F_{d, 10}=F_{\text {drag }}+F_{\text {thruster, }}$, where

$$
F_{\text {drag }}=m_{0} \times\left[\begin{array}{c}
2.15 \times 10^{-7} \sin (0.00114 t) \\
0.15 \times 10^{-4} \sin (0.00114 t)-2.35 \times 10^{-4} \\
0.20 \times 10^{-5} \sin (0.00114 t)-2.05 \times 10^{-5}
\end{array}\right] N \text {. }
$$

The resolution and the maximum value of the thruster are $10^{-6} \mathrm{~N}$ and $0.4 \mathrm{~N}$. Besides, a zero mean white noise with mean squared error being $10^{-8} \mathrm{~N}$ is added as the stochastic disturbance.

For the relative position motion between TM1 and TM2, define $F_{d, 12}=F_{\text {elec }}+F_{\text {actuator }}$, where

$$
F_{\text {elec }}=m_{1} \times\left[\begin{array}{c}
1 \times 10^{-6} \sin \left(0.04 t+\frac{\pi}{3}\right) \\
1 \times 10^{-6} \sin (0.05 t) \\
1 \times 10^{-6} \sin \left(0.05 t+\frac{\pi}{2}\right)
\end{array}\right] N \text {. }
$$


TABle 1: Parameters of satellite used in the simulation.

\begin{tabular}{|c|c|c|c|c|}
\hline Symbols & \multicolumn{4}{|c|}{ Parameters } \\
\hline$m_{0}$ & \multicolumn{4}{|c|}{$145 \mathrm{~kg}$} \\
\hline$m_{1}$ & \multicolumn{4}{|c|}{$1 \mathrm{~kg}$} \\
\hline$m_{2}$ & \multicolumn{4}{|c|}{$1 \mathrm{~kg}$} \\
\hline$\omega_{0}$ & \multicolumn{4}{|c|}{$0.0011569 \mathrm{rad} / \mathrm{s}$} \\
\hline$\tilde{\rho}_{\max }$ & \multicolumn{4}{|c|}{$0.1 \mathrm{~m}$} \\
\hline$\dot{\tilde{\rho}}_{\max }$ & \multicolumn{4}{|c|}{$0.1 \mathrm{~m} / \mathrm{s}$} \\
\hline & 1 & 0.0390 & $0.039]$ & \\
\hline$K_{t}$ & 0.03 & $\begin{array}{ll}1 & 0\end{array}$ & 0.039 & $\times 10^{-6} \mathrm{~N} / \mathrm{m}$ \\
\hline & 0.03 & 0.039 & $1]$ & \\
\hline$D_{t}$ & \multicolumn{4}{|c|}{$1.4 \times 10^{-11} I_{3} \mathrm{~N} /(\mathrm{m} / \mathrm{s})$} \\
\hline
\end{tabular}

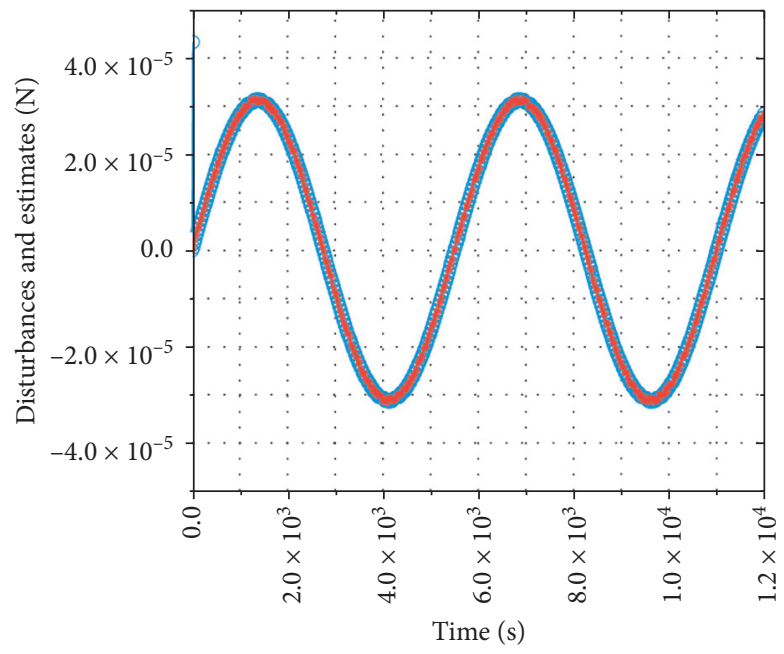

$\multimap \hat{\xi}_{1,1}$

$\longrightarrow \xi_{1,1}$

(a)

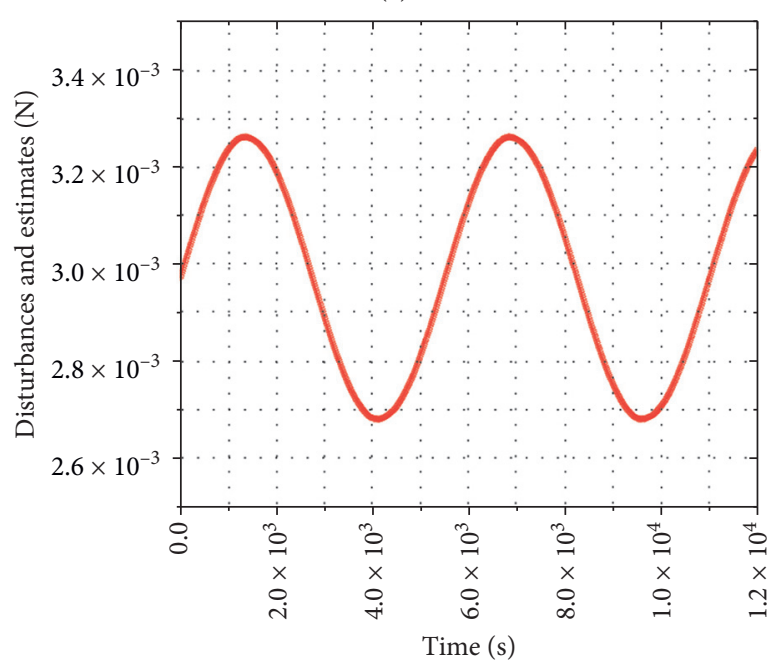

$\smile \hat{\xi}_{1,3}$

$\longrightarrow \xi_{1,3}$

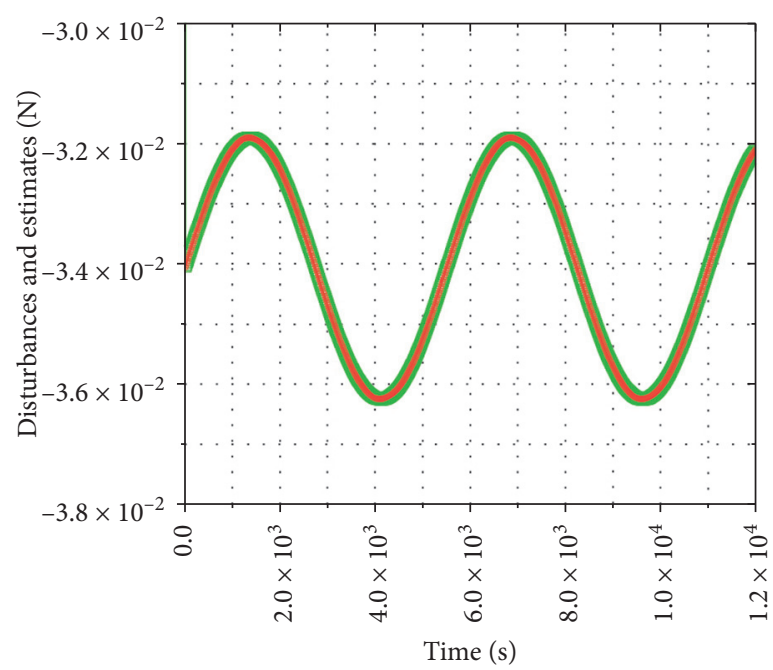

$\square \hat{\xi}_{1,2}$

$\longrightarrow \xi_{1,2}$

(b)

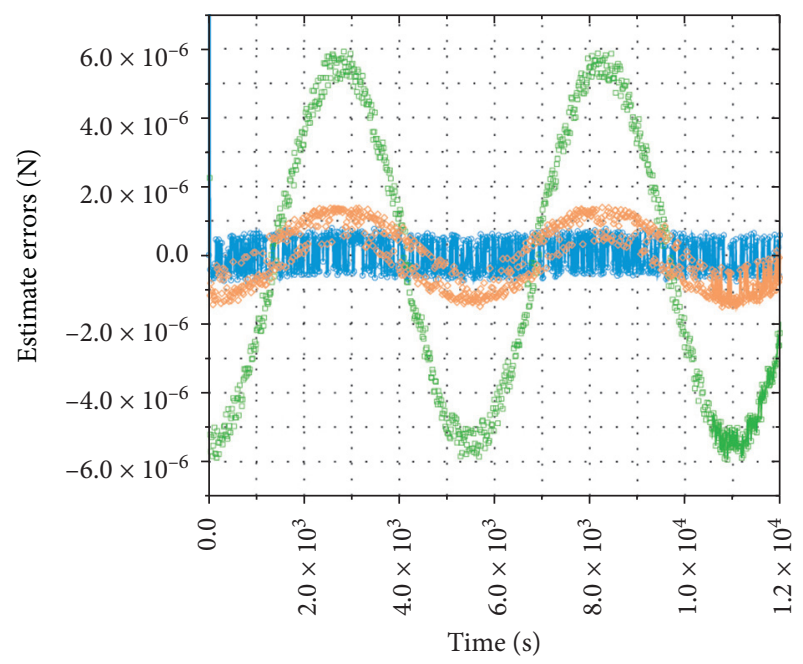

$-\tilde{\xi}_{1,1}$

$-\tilde{\xi}_{1,2}$

$\simeq \widetilde{\xi}_{1,3}$

(c)

(d)

Figure 4: Estimation of the disturbances $\xi_{1}(t)$ by the ESMO. 


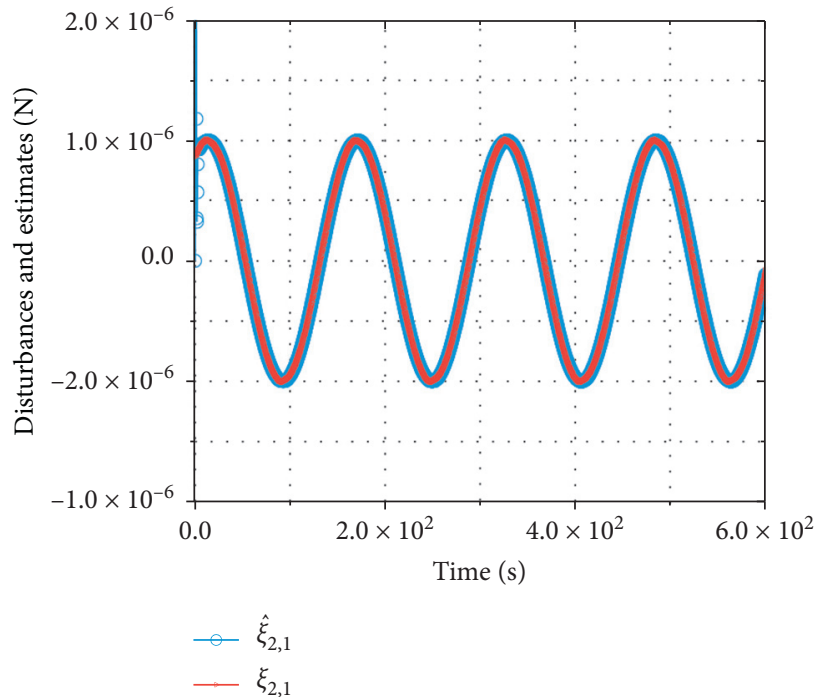

(a)

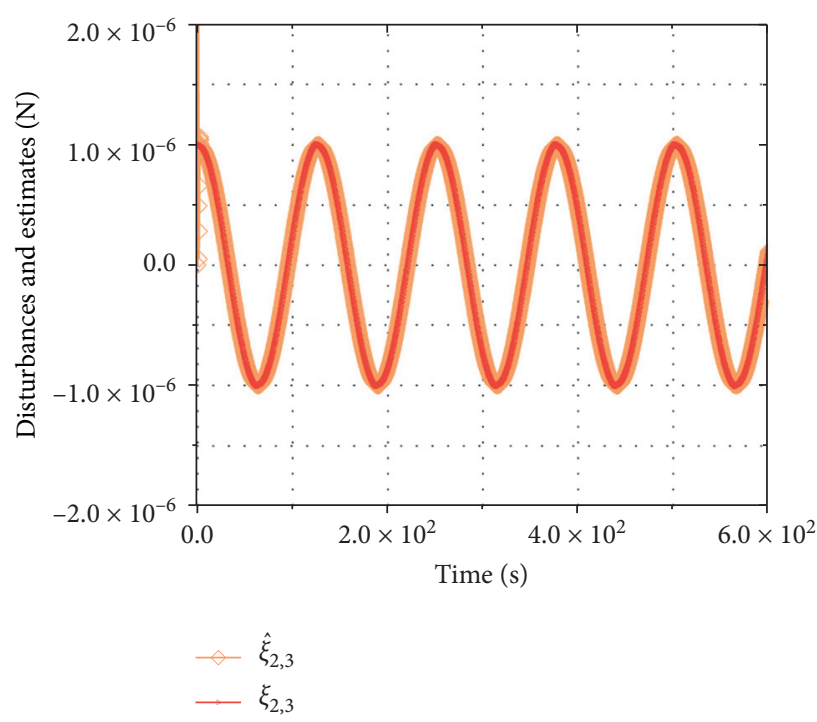

(c)

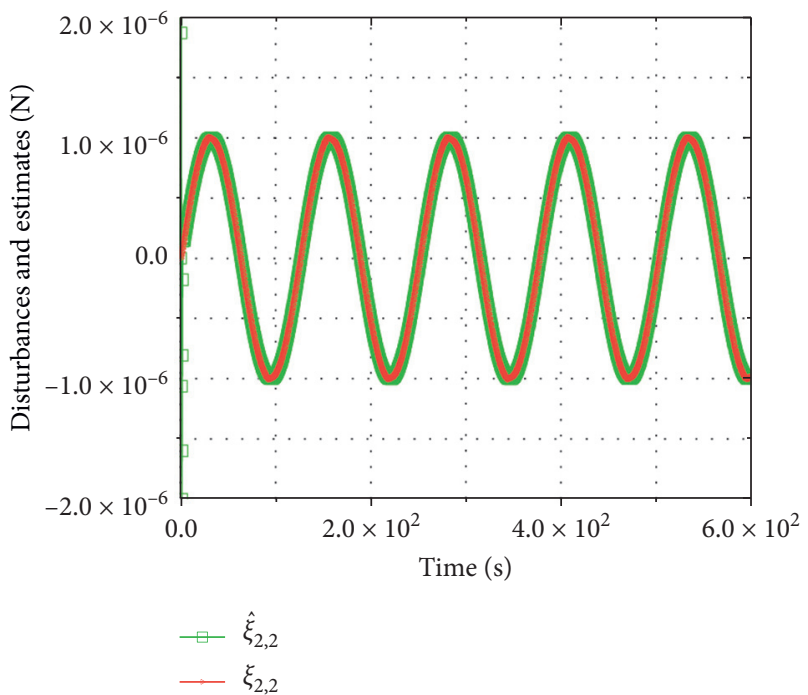

(b)
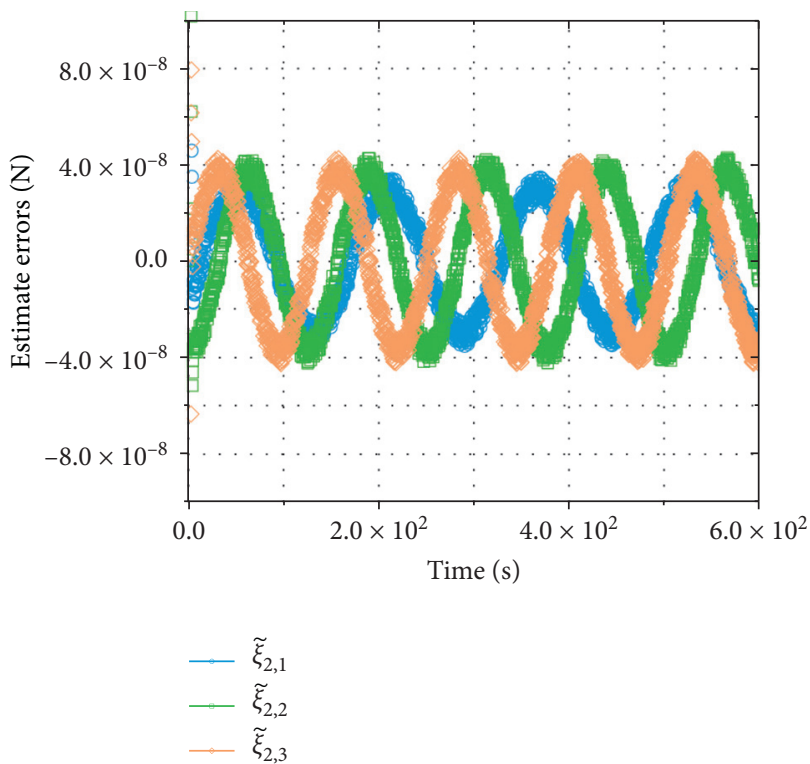

(d)

Figure 5: Estimation of the disturbances $\xi_{2}(t)$ by the ESMO.

The resolution and the maximum value of the thruster are $10^{-7} \mathrm{~N}$ and $60 \times 10^{-6} \mathrm{~N}$. Similarly, a zero mean white noise with mean squared error being $10^{-8} \mathrm{~N}$ is added as the stochastic disturbance.

The simulation results are shown in Figures $4-10$ as follows. Define the estimated error $\widetilde{\xi}_{i}(t)=\widehat{\xi}_{i}(t)-\xi_{i}(t)(i=1$, 2 ), as shown in Figures 4 and 5 , and $\xi_{i}(t)$ can be estimated precisely by the ESMO. From Figures $6-11$, the relative motion variables $\widetilde{\rho}_{10}(t), \dot{\tilde{\rho}}_{10}(t), \tilde{\rho}_{12}(t)$, and $\dot{\tilde{\rho}}_{12}(t)$ could be estimated well by the ESMO. In addition, the stable control accuracy of the composite control approach has achieved $10^{-7}$ or $10^{-8}$, which shows that the developed extended sliding mode observer method and tube-based model predictive control law are effective. 


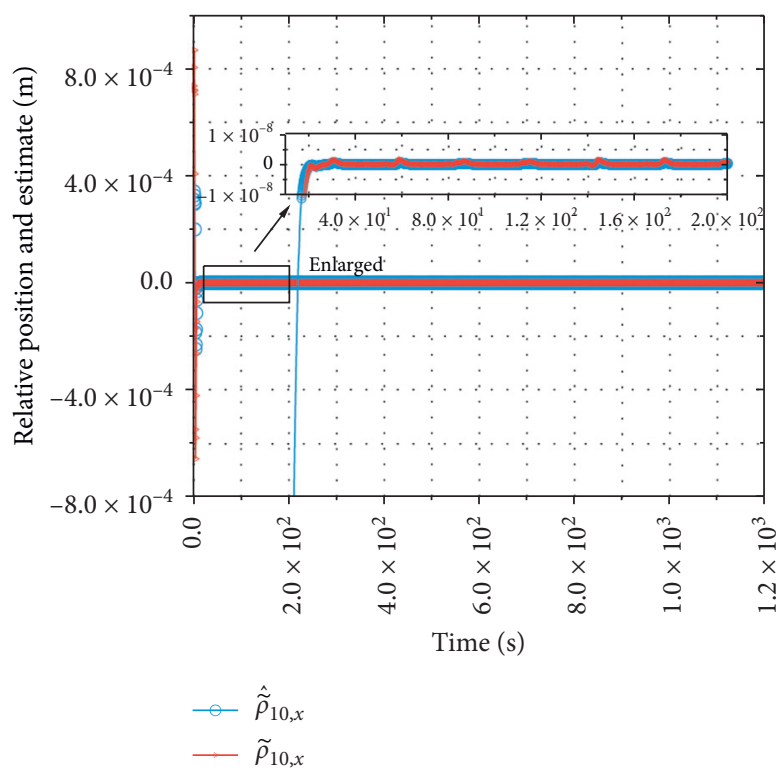

(a)

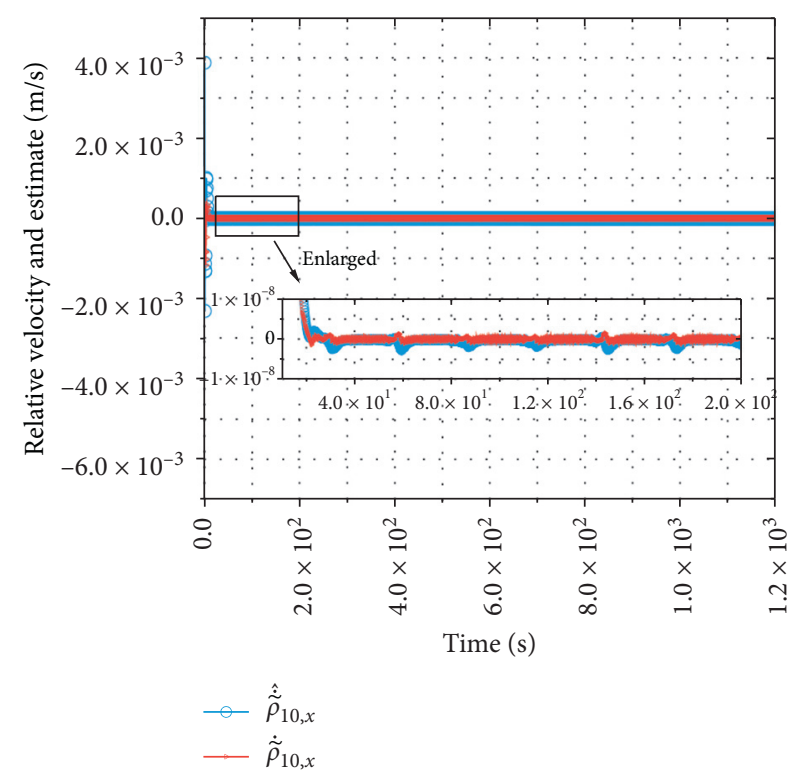

(b)

FIGURE 6: Simulated relative positions $\widetilde{\rho}_{10, x}(t), \dot{\tilde{\rho}}_{10, x}(t)$ and their estimations between TM1 and the satellite.

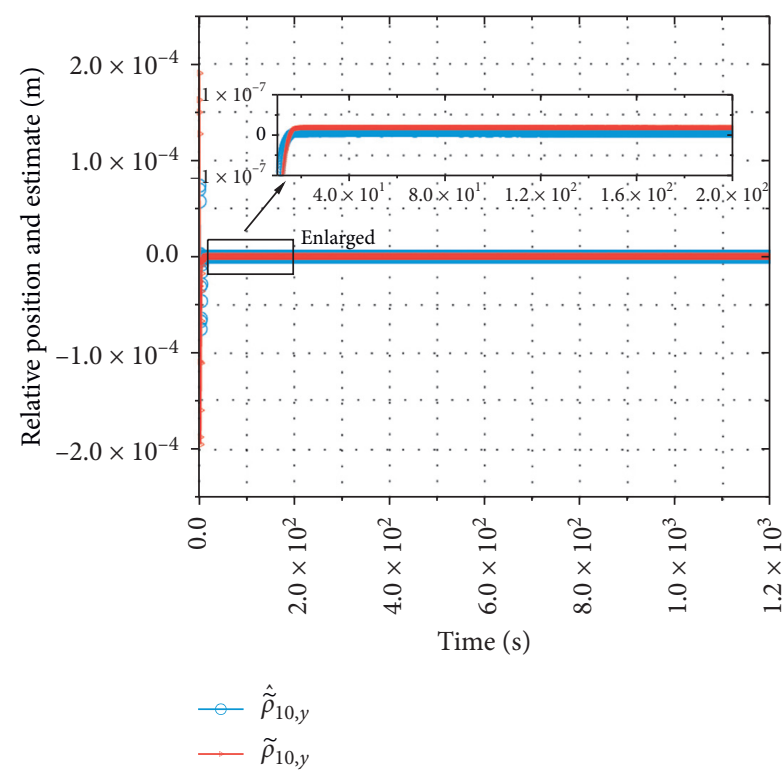

(a)

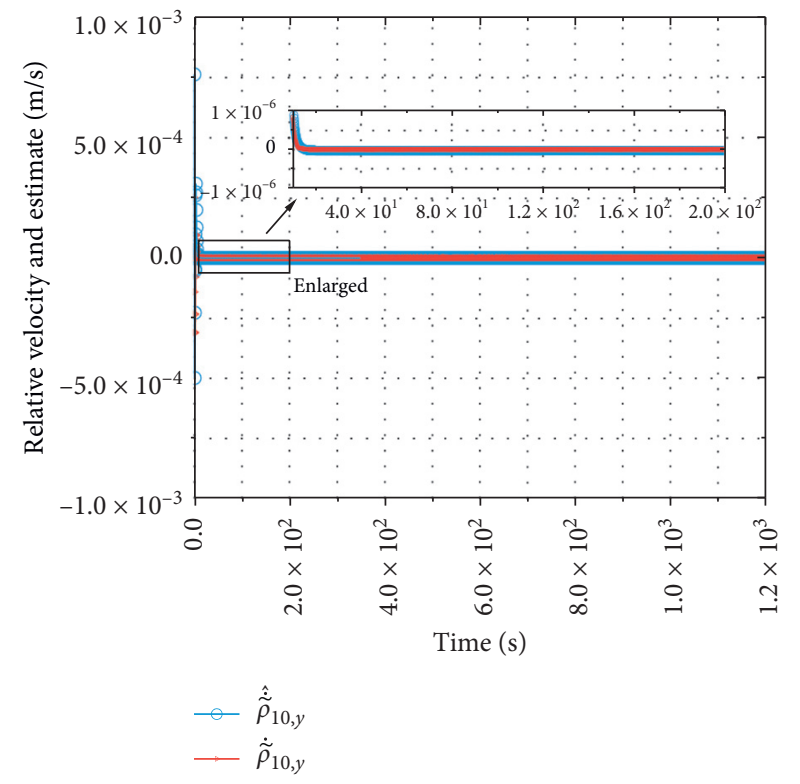

(b)

FIGURE 7: Simulated relative positions $\widetilde{\rho}_{10, y}(t), \dot{\tilde{\rho}}_{10, y}(t)$ and their estimations between TM1 and the satellite. 


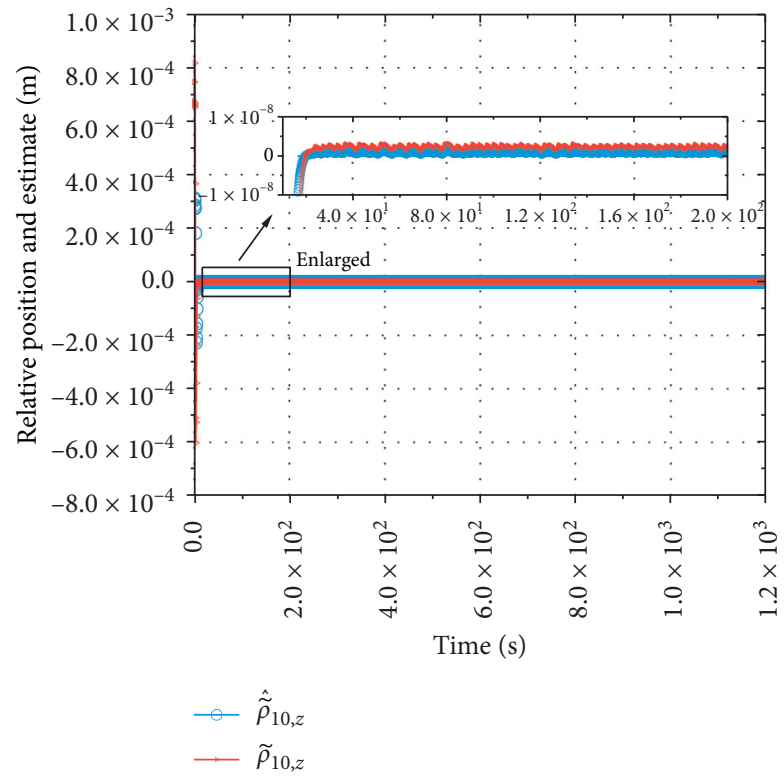

(a)

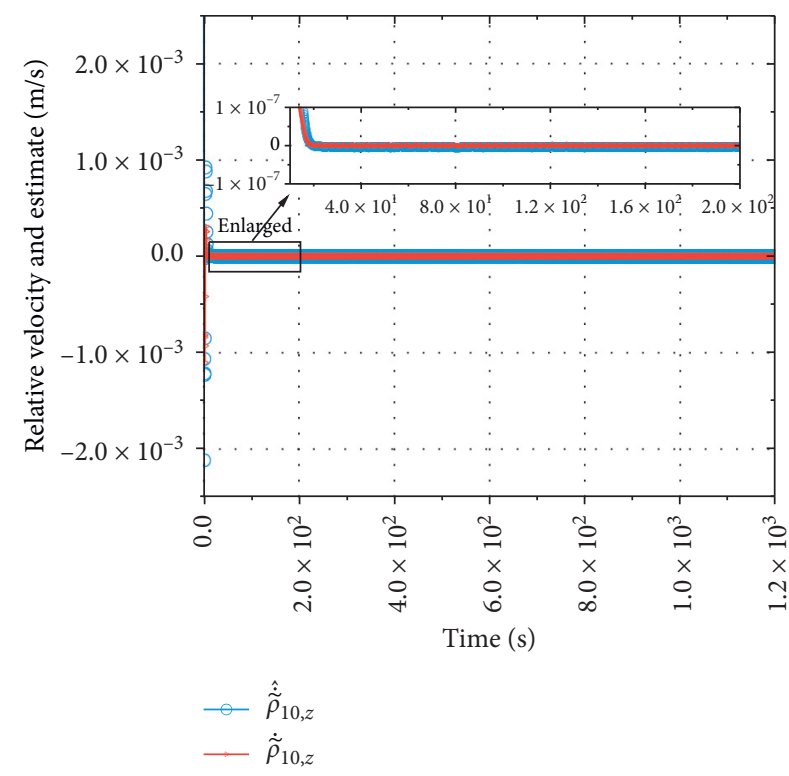

(b)

FIGURE 8: Simulated relative positions $\widetilde{\rho}_{10, z}(t), \dot{\tilde{\rho}}_{10, z}(t)$ and their estimations between TM1 and the satellite.
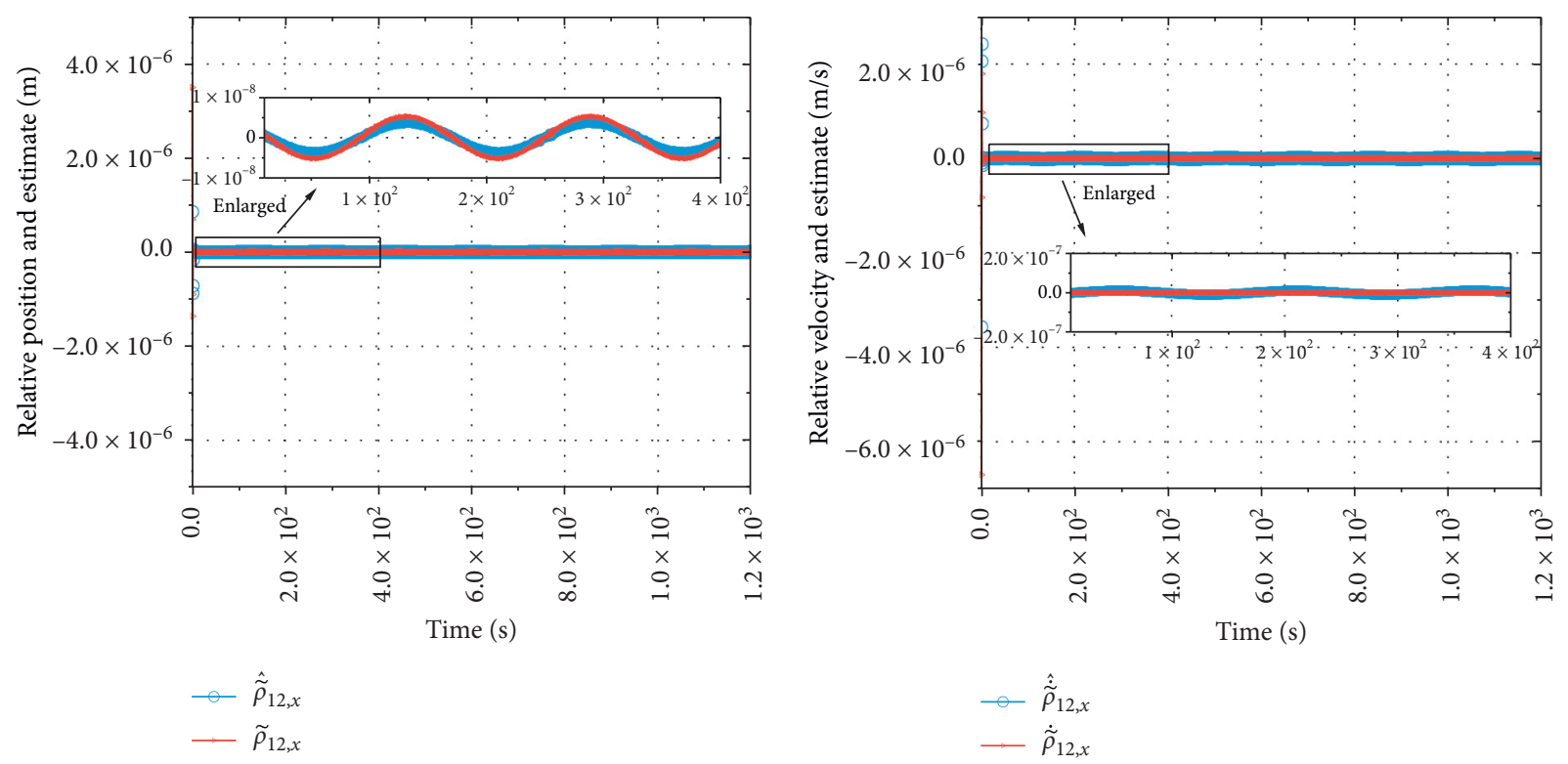

(a)

(b)

FIGURE 9: Simulated relative positions $\tilde{\rho}_{12, x}(t), \dot{\tilde{\rho}}_{12, x}(t)$ and their estimations between TM1 and TM2. 


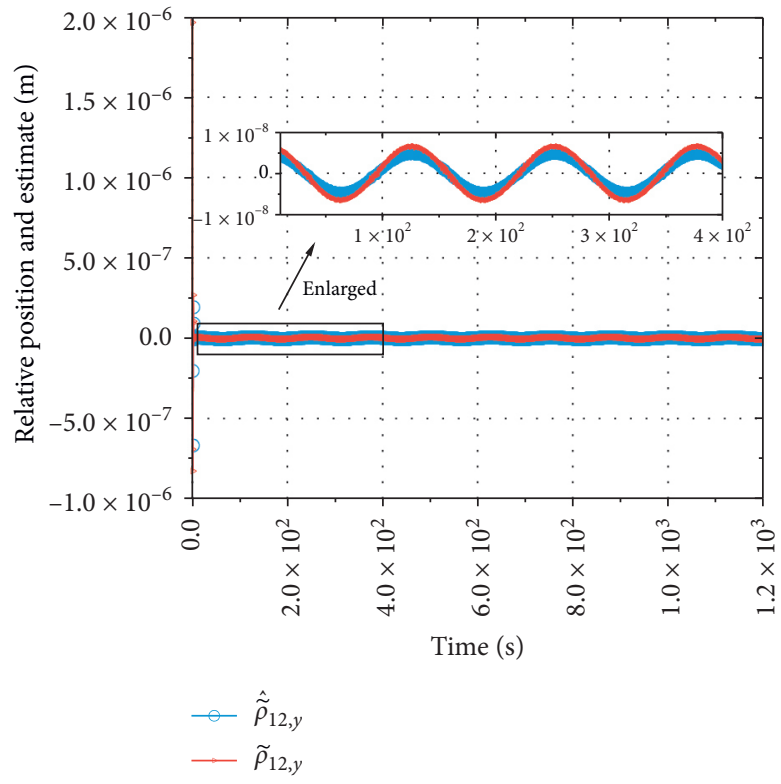

(a)

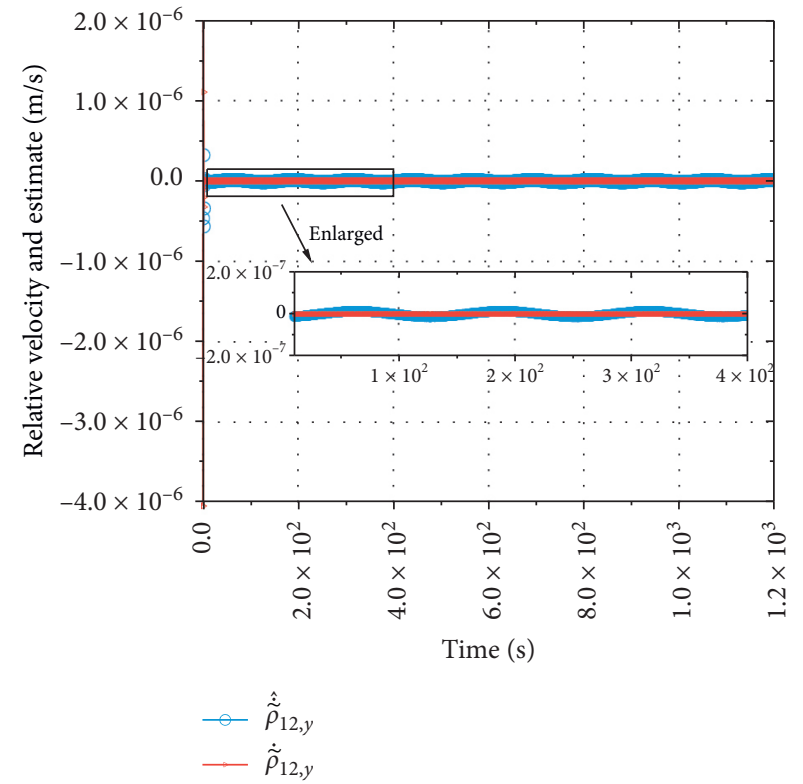

(b)

FIGURE 10: Simulated relative positions $\widetilde{\rho}_{12, y}(t), \dot{\tilde{\rho}}_{12, y}(t)$ and their estimations between TM1 and TM2.

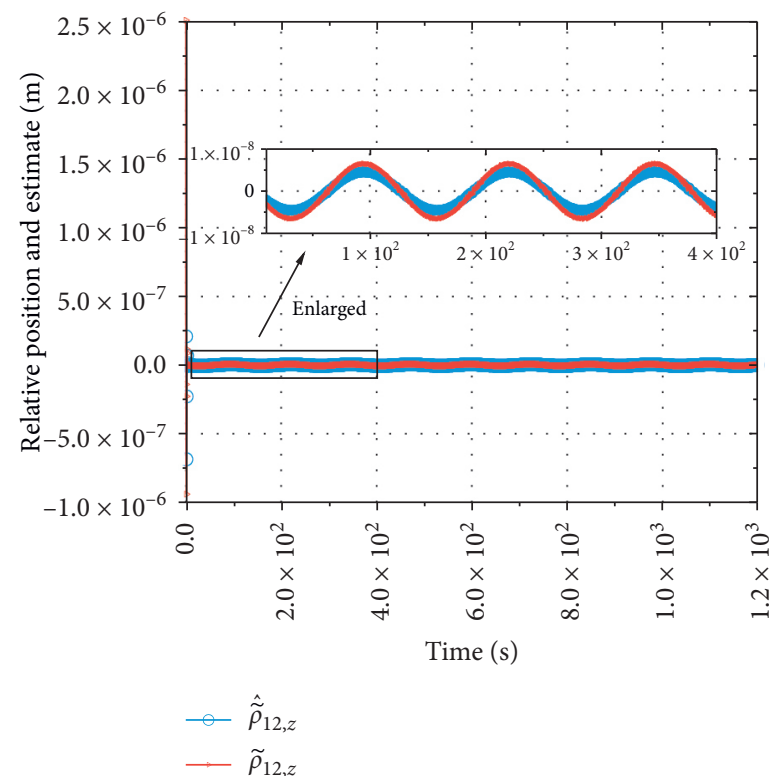

(a)

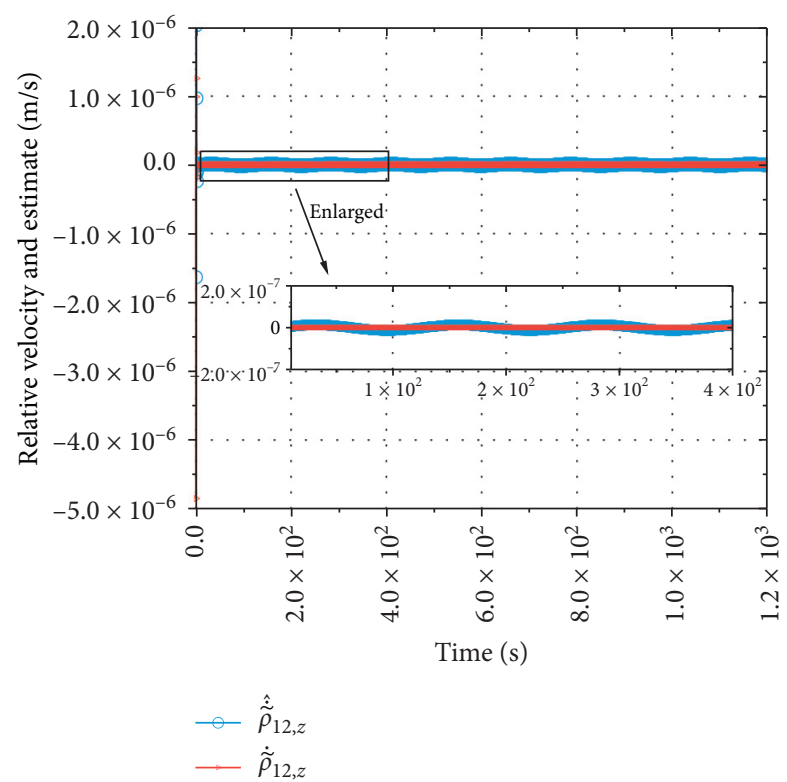

(b)

FIGURE 11: Simulated relative positions $\tilde{\rho}_{12, z}(t), \dot{\tilde{\rho}}_{12, z}(t)$ and their estimations between TM1 and TM2. 


\section{Conclusions}

This paper has considered the relative position control of the drag-free satellite with double cube test-masses in the presence of external disturbance, additive stochastic disturbances, actuator quantization error, actuator saturation, and state constraints. An extended sliding mode observer method is adopted to estimate the state vector and external disturbance, based on which a tube-based robust model predictive control scheme is developed. The designed control method can not only cope with the constraints of control and state but also attenuate the effect of additive stochastic noises. Future work will be focused on the consideration of relative attitude dynamics between the test-masses and the satellite.

\section{Data Availability}

The data used to support the findings of this study are available from the corresponding author upon request.

\section{Conflicts of Interest}

The authors declare that there are no conflicts of interest regarding the publication of this paper.

\section{Acknowledgments}

This work was supported by the National Natural Science Foundation of China (61833009, 11972130, and 61690212) and Guangdong Major Project of Basic and Applied Basic Research (Grant no. 2019B030302001).

\section{References}

[1] B. Lange, "The drag-free satellite," AIAA Journal, vol. 2, no. 9, pp. 1590-1606, 1964.

[2] E. Hardy, M. S. Rodrigues, P. Touboul et al., "Testing the equivalence principle in space: the MICROSCOPE mission," in Proceedings of the 42nd COSPAR Scientific Assembly, Pasadena, CA, USA, 2018.

[3] A. N. Marchenko, D. A. Marchenko, and A. N. Lopushansky, "Gravity field models derived from the second degree radial derivatives of the GOCE mission: a case study," Annals of Geophysics, vol. 59, no. 6, p. 0649, 2017.

[4] P. Amaro-Seoane, H. Audley, S. Babak et al., "Laser interferometer space antenna," 2017, https://arxiv.org/abs/1702. 00786.

[5] J. Conklin and A. N. Nguyen, "Drag-free control and drag force recovery of small satellites," in Proceedings of the 2017 Annual AIAA/USU Conference on Small Satellites, Logan, UT, USA, 2017.

[6] A. N. Nguyen and J. W. Conklin, "Three-axis drag-free control and drag force recovery of a single-thruster small satellite," Journal of Spacecraft and Rockets, vol. 52, no. 6, pp. 1640-1650, 2015.

[7] C. F. Everitt, D. DeBra, B. Parkinson et al., "Gravity probe B: final results of a space experiment to test general relativity," Physical Review Letters, vol. 106, no. 22, p. 221101, 2011.

[8] P. Prieur, T. Lienart, M. Rodrigues et al., "MICROSCOPE mission: on-orbit assessment of the drag-free and attitude control system," in Proceedings of the 31st International
Symposium on Space Technology and Science (ISTS 2017), Kobe, Japan, 2017.

[9] E. Canuto, "Drag-free and attitude control for the GOCE satellite," Automatica, vol. 44, no. 7, pp. 1766-1780, 2008.

[10] E. Canuto, L. Colangelo, M. Lotufo, and S. Dionisio, "Satelliteto-satellite attitude control of a long-distance spacecraft formation for the next generation gravity mission," European Journal of Control, vol. 25, pp. 1-16, 2015.

[11] B. D. Loomis, R. S. Nerem, and S. B. Luthcke, "Simulation study of a follow-on gravity mission to GRACE," Journal of Geodesy, vol. 86, no. 5, pp. 319-335, 2012.

[12] J. Baker, J. Bellovary, P. L. Bender et al., "The laser interferometer space antenna: unveiling the Millihertz gravitational wave sky," 2019, https://arxiv.org/abs/1907.06482.

[13] M. Armano, H. Audley, J. Baird et al., "LISA Pathfinder platform stability and drag-free performance," Physical Review D, vol. 99, no. 8, Article ID 082001, 2019.

[14] Z. Dang and Y. Zhang, "Relative position and attitude estimation for inner-formation gravity measurement satellite system," Acta Astronautica, vol. 69, no. 7-8, pp. 514-525, 2011.

[15] J. Luo, L. S. Chen, H. Z. Duan et al., "TianQin: a space-borne gravitational wave detector," Classical and Quantum Gravity, vol. 33, no. 3, Article ID 035010, 2016.

[16] G. D. Racca and P. W. McNamara, "The LISA pathfinder mission," Space Science Reviews, vol. 151, no. 1-3, pp. 159-181, 2010.

[17] L. Ji, J. Xiang, and K. Liu, "Model predictive technics based three-axis stabilization control of a drag-free satellite," in Proceedings of the 2010 3rd International Conference on Computer Science and Information Technology, IEEE, Chengdu, China, 2010.

[18] W. Fichter, P. Gath, S. Vitale, and D. Bortoluzzi, "LISA pathfinder drag-free control and system implications," Classical and Quantum Gravity, vol. 22, no. 10, pp. S139-S148, 2005.

[19] L. Pettazzi, A. Lanzon, S. Theil, and A. E. Finzi, "Design of robust drag-free controllers with given structure," Journal of Guidance, Control, and Dynamics, vol. 32, no. 5, pp. 16091621, 2009.

[20] Z. Dang and Y. Zhang, "Formation control using $\mu$-synthesis for inner-formation gravity measurement satellite system," Advances in Space Research, vol. 49, no. 10, pp. 1487-1505, 2012.

[21] E. Canuto and L. Massotti, "All-propulsion design of the dragfree and attitude control of the European satellite GOCE," Acta Astronautica, vol. 64, no. 2-3, pp. 325-344, 2009.

[22] S.-F. Wu and D. Fertin, "Spacecraft drag-free attitude control system design with quantitative feedback theory," Acta Astronautica, vol. 62, no. 12, pp. 668-682, 2008.

[23] W. H. Chen, J. Yang, L. Guo et al., "Disturbance-observerbased control and related methods-an overview," IEEE Transactions on Industrial Electronics, vol. 63, no. 2, pp. 1083-1095, 2015.

[24] Q. Hou, S. Ding, and X. Yu, “Composite super-twisting sliding mode control design for PMSM speed regulation problem based on a novel disturbance observer," IEEE Transactions on Energy Conversion, p. 1, 2020.

[25] S. Ding, W.-H. Chen, K. Mei, and D. J. Murray-Smith, "Disturbance observer design for nonlinear systems represented by input-output models," IEEE Transactions on Industrial Electronics, vol. 67, no. 2, pp. 1222-1232, 2020.

[26] X. Zhao, X. Ma, and X. Ma, "A novel disturbance observer for multiagent tracking control with matched and unmatched 
uncertainties," Mathematical Problems in Engineering, vol. 2019, Article ID 9765353, 9 pages, 2019.

[27] J. Yan, H. Wang, S. Huang, and Y. Lan, "Disturbance observer-based backstepping control of PMSM for the mine traction electric locomotive," Mathematical Problems in Engineering, vol. 2018, Article ID 7253210, 10 pages, 2018.

[28] X. Zhang, J. Gao, W. Zhang, T. Zeng, and L. Ye, "Distributed formation control for multiple quadrotor based on multiagent theory and disturbance observer," Mathematical Problems in Engineering, vol. 2019, Article ID 7234969, 11 pages, 2019.

[29] L. Liu, X. Song, Z. Fu, and S. Song, "Disturbance observerbased input-output finite-time control of a class of nonlinear systems," Mathematical Problems in Engineering, vol. 2017, Article ID 7202584, 7 pages, 2017.

[30] H. Liang, X. Guo, Y. Pan, and T. Huang, "Event-triggered fuzzy bipartite tracking control for network systems based on distributed reduced-order observers (revised manuscript of TFS-2019-1049)," IEEE Transactions on Fuzzy Systems, p. 1, 2020.

[31] P. Du, Y. Pan, H. Li, and H.-K. Lam, "Nonsingular finite-time event-triggered fuzzy control for large-scale nonlinear systems," IEEE Transactions on Fuzzy Systems, p. 1, 2020.

[32] Z. Zhu, Y. Pan, Q. Zhou, and C. Lu, "Event-triggered adaptive fuzzy control for stochastic nonlinear systems with unmeasured states and unknown backlash-like hysteresis," IEEE Transactions on Fuzzy Systems, p. 1, 2020.

[33] Q. Zhou, S. Zhao, H. Li, R. Lu, and C. Wu, "Adaptive neural network tracking control for robotic manipulators with dead zone," IEEE Transactions on Neural Networks and Learning Systems, vol. 30, no. 12, pp. 3611-3620, 2019.

[34] J. Han, "From PID to active disturbance rejection control," IEEE Transactions on Industrial Electronics, vol. 56, no. 3, pp. 900-906, 2009.

[35] C. Zhong, Z. Chen, and Y. Guo, "Attitude control for flexible spacecraft with disturbance rejection," IEEE Transactions on Aerospace and Electronic Systems, vol. 53, no. 1, pp. 101-110, 2017.

[36] W. Wang, H. Liang, Y. Pan, and T. Li, "Prescribed performance adaptive fuzzy containment control for nonlinear multiagent systems using disturbance observer," IEEE Transactions on Cybernetics, vol. 50, no. 9, pp. 3879-3891, 2020.

[37] G. Dong, L. Cao, D. Yao et al., "Adaptive attitude control for multi-MUAVs with output dead-zone and actuator fault," IEEE/CAA Journal of Automatica Sinica, 2020.

[38] G. Lin, H. Li, H. Ma et al., "Human-in-the-loop consensus control for nonlinear multi-agent systems with actuator faults," IEEE/CAA Journal of Automatica Sinica, 2020.

[39] H. Ma, H. Li, R. Lu et al., "Adaptive event-triggered control for a class of nonlinear systems with periodic disturbances," Science China Information Sciences, vol. 63, no. 5, pp. 1-15, 2020.

[40] E. Canuto, A. Molano, and L. Massotti, "Drag-free control of the GOCE satellite: noise and observer design," IEEE Transactions on Control Systems Technology, vol. 18, no. 2, pp. 501-509, 2009.

[41] H. Li, Y. Bai, M. Hu et al., "A novel controller design for the next generation space electrostatic accelerometer based on disturbance observation and rejection," Sensors, vol. 17, no. 1, p. 21, 2017.

[42] L. Cao, B. Xiao, and M. Golestani, "Robust fixed-time attitude stabilization control of flexible spacecraft with actuator uncertainty," Nonlinear Dynamics, vol. 100, no. 3, pp. 2505-2519, 2020.

[43] B. Xiao, L. Cao, S. Xu, and L. Liu, "Robust tracking control of robot manipulators with actuator faults and joint velocity measurement uncertainty," IEEE/ASME Transactions on Mechatronics, vol. 25, no. 3, pp. 1354-1365, 2020.

[44] R. Yang and W. X. Zheng, "Output-based event-triggered predictive control for networked control systems," IEEE Transactions on Industrial Electronics, vol. 67, no. 12, pp. 10631-10640, 2020.

[45] R. Yang, W. X. Zheng, and Y. Yu, "Event-triggered sliding mode control of discrete-time two-dimensional systems in Roesser model," Automatica, vol. 114, Article ID 108813, 2020.

[46] Y. Zhu and W. X. Zheng, "Multiple Lyapunov functions analysis approach for discrete-time-switched piecewise-affine systems under dwell-time constraints," IEEE Transactions on Automatic Control, vol. 65, no. 5, pp. 2177-2184, 2020.

[47] Y. Zhu, W. X. Zheng, and D. Zhou, "Quasi-synchronization of discrete-time lure-type switched systems with parameter mismatches and relaxed PDT constraints," IEEE Transactions on Cybernetics, vol. 50, no. 5, pp. 2026-2037, 2020.

[48] Y. Zhu and W. X. Zheng, "Observer-based control for cyberphysical systems with periodic DoS attacks via a cyclic switching strategy," IEEE Transactions on Automatic Control, vol. 65 , no. 8 , pp. 3714-3721, 2020.

[49] L. Liu, W. X. Zheng, and S. Ding, "An adaptive SOSM controller design by using a sliding-mode-based filter and its application to buck converter," IEEE Transactions on Circuits and Systems I: Regular Papers, vol. 67, no. 7, pp. 2409-2418, 2020.

[50] C. Zhang, J. He, L. Duan, and Q. Kang, "Design of an active disturbance rejection control for drag-free satellite," Microgravity Science and Technology, vol. 31, no. 1, pp. 31-48, 2019.

[51] E. Canuto, W. Acuna-Bravo, A. Molano-Jimenez, and C. Perez Montenegro, "Embedded model control calls for disturbance modeling and rejection," ISA Transactions, vol. 51, no. 5, pp. 584-595, 2012.

[52] S. V. Raković, "Model predictive control: classical, robust, and stochastic," IEEE Control Systems Magazine, vol. 36, no. 6, pp. 102-105, 2016.

[53] Z. Dang, "Modeling and controller design of inner-formation flying system with two proof-masses," Aerospace Science and Technology, vol. 30, no. 1, pp. 8-17, 2013.

[54] J. Zhou, L. Liu, and Z. Wang, "Modeling and analysis of ultralow frequency dynamics of drag-free satellites," Microgravity Science and Technology, vol. 31, no. 2, pp. 151-160, 2019.

[55] J. Zhang, P. Shi, and W. Lin, "Extended sliding mode observer based control for Markovian jump linear systems with disturbances," Automatica, vol. 70, pp. 140-147, 2016.

[56] Y. Shtessel, C. Edwards, L. Fridman et al., Sliding Mode Control and Observation, Springer, Berlin, Germany, 2014.

[57] J. B. Rawlings and D. Q. Mayne, Model Predictive Control: Theory and Design, Nob Hill Pub, Madison, WI, USA, 2009. 